\title{
DÜBLIN
}

Technological University Dublin

ARROW@TU Dublin

2007-1

\section{Hologram-based Humidity Indicator for Domestic and Packaging Applications}

\author{
Izabela Naydenova \\ Technological University Dublin, izabela.naydenova@tudublin.ie \\ Raghavendra Jallapuram \\ Technological University Dublin \\ Vincent Toal \\ Technological University Dublin, vincent.toal@tudublin.ie
}

See next page for additional authors

Follow this and additional works at: https://arrow.tudublin.ie/cieocon2

Part of the Condensed Matter Physics Commons, and the Optics Commons

\section{Recommended Citation}

Naydenova, I., Jallapuram, R. \& Toal, V. (2007). Hologram-based humidity indicator for domestic and packaging applications. SPIE Proceedings, vol. 6528, 652811. doi:10.1117/12.716242

This Conference Paper is brought to you for free and open access by the Centre for Industrial and Engineering Optics at ARROW@TU Dublin. It has been accepted for inclusion in Conference Papers by an authorized administrator of ARROW@TU Dublin. For more information, please contact arrow.admin@tudublin.ie, aisling.coyne@tudublin.ie, gerard.connolly@tudublin.ie.

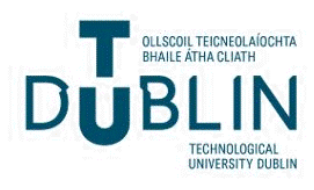




\section{Authors}

Izabela Naydenova, Raghavendra Jallapuram, Vincent Toal, and Suzanne Martin 
Dublin Institute of Technology

ARROW@DIT

2007-01-01

\section{Hologram-based humidity indicator for domestic and packaging applications}

Izabela Naydenova

Dublin Institute of Technology, izabela.naydenova@dit.ie

Raghavendra Jallapuram

Dublin Institute of Technology

Vincent Toal

Dublin Institute of Technology, vincent.toal@dit.ie

Suzanne Martin

Dublin Institute of Technology, suzanne.martin@dit.ie

\section{Recommended Citation}

I. Naydenova, R. Jallapuram, V. Toal and S. Martin, "Hologram-based humidity indicator for domestic and packaging applications", SPIE proceedings, V 6528, 652811, 2007.doi:10.1117/12.716242

This Conference Paper is brought to you for free and open access by the Centre for Industrial and Engineering Optics at ARROW@DIT. It has been accepted for inclusion in Articles by an authorized administrator of ARROW@DIT. For more information, please contact

yvonne.desmond@dit.ie, arrow.admin@dit.ie. 


\title{
Hologram-based humidity indicator for domestic and packaging applications
}

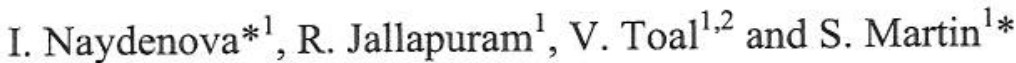 \\ ${ }^{1}$ Centre for Industrial and Engineering Optics, Kevin Street, DIT, Dublin8, Ireland \\ ${ }^{2}$ School of Physics, Kevin Street, DIT, Dublin 8, Ireland
}

\begin{abstract}
A passive indicator for Relative Humidity based on a photopolymer recorded hologram has been developed. The indicator works on the principle that the wavelength reconstructed by a reflection hologram is dependent on the spacing between the fringe planes of the hologram. As the Relative Humidity changes, the hologram swells or shrinks and the apparent colour of the reconstructed image changes.

The response time, operational range and reversibility of the indicator have been studied in a controlled humidity environment. Response times from less than a minute to tens of minutes depending on the holograms physical properties have been measured. Indicators with different operational ranges have been designed. For some indicators the operational range extends from $10 \% \mathrm{RH}$ to $80 \% \mathrm{RH}$.
\end{abstract}

The indicator is completely reversible in its present formulation. It could have an extensive market in industrial monitoring, food packaging and household applications.

Keywords: holographic sensors, relative humidity, humidity indicator, reflection hologram, photopolymer

\section{INTRODUCTION}

At present relative humidity may be measured by means of a humidity meter that is a device that detects the change of a physical property of a thin sensing film such as capacitance, resistivity, thermal conductivity, etc in response to the change in the relative humidity of the environment. However, such devices need a power source, or to need be connected to a power source in order to detect this change and they are relatively complex, expensive and bulky devices which include electronic components. A much simplified approach for measuring relative humidity utilises a card containing spots of silica gel that indicate a change in relative humidity by a colour change. However, such devices often have a slow response time and limited accuracy and are not suitable for use with foodstuffs. The specially designed photopolymer holograms discussed here could prove to be good candidates for fabrication of more accurate, faster response humidity indicators and suitable for use in food packaging. At the same time when produced on a large scale their price could be comparable with that of the silica gel spot cards.

Holographic sensors for detection of different types of analytes have been attracting significant attention in recent years ${ }^{1-}$ 3. For example ${ }^{3}$ a holographic sensor based on pre-recorded volume hologram was developed by Smart Holograms Ltd. A hologram is disposed throughout a support medium which is analyte sensitive. The process of detection consists of variation of one or more optical characteristics of the hologram due to variation(s) in the hologram support medium caused by the reaction of the analyte in liquid form with a substance disposed throughout the sensor.

In the present paper we report the results from the fabrication and the characterisation of a highly sensitive hologram that operates in a similar manner but that doesn't require immersion in liquid and changes colour when exposed to a change in relative humidity.

*izabela.naydenova@dit.ie; phone 0035314027925; fax: 0035314027901; www.ieo.dit.ie

*suzanne.martin@dit.ie; phone 0035314024613; fax:0035314027901; www.ieo.dit.ie

Nanosensors, Microsensors, and Biosensors and Systems 2007, edited by Vijay K. Varadan, Proc. of SPIE Vol. 6528, 652811, (2007) · 0277-786X/07/\$18 · doi: 10.1117/12.716242 


\section{THEORY}

\subsection{Holographic sensors}

In general, the holographic sensor contains a holographic optical element that is disposed throughout a support medium. The holographic optical element is fabricated ${ }^{4}$ by exposing a suitable photosensitive material to the optical interference pattern produced when two coherent light beams, usually from a laser, intersect. The material records the variation in light intensity as a variation in refractive index, absorption or thickness, and a corresponding holographic optical element is produced. There is a large variety of holographic optical elements that can be produced and used to design a detection system: volume or surface relief holograms, transmission or reflection holograms, phase or amplitude holograms.

The humidity indicator reported in the present paper is designed on the basis of a reflection volume phase hologram and a more detailed description of this particular type of holographic optical element is presented in the following section.

\subsection{Reflection hologram}

The recording and replay of a reflection hologram can be explained with the help of Fig. 1. In this geometry of recording the two coherent beams are incident from the two opposite sides of the recording medium. If both light beams are plane waves, the result is a simple diffraction grating whose spatial period depends on the angle between the two beams and is determined by the following relation ${ }^{5}$ :

$$
\Lambda=\frac{\lambda}{2 n_{0} \sin \theta}
$$

where $\theta$ is the half of the angle between the two recording beam inside the recording medium, $\lambda$ is the wavelength of the recording light, $n_{0}$ is the refractive index of the recording medium and $\Lambda$ is the fringe spacing.

When the beams have the same incident angles the interference fringes are parallel to the surface of the recording medium and in this case an unslanted reflection grating is recorded. The incident angles of the two recording beams could also be different and then the recorded fringes would be at an angle with respect to the recording medium surface and the recorded grating would be slanted.

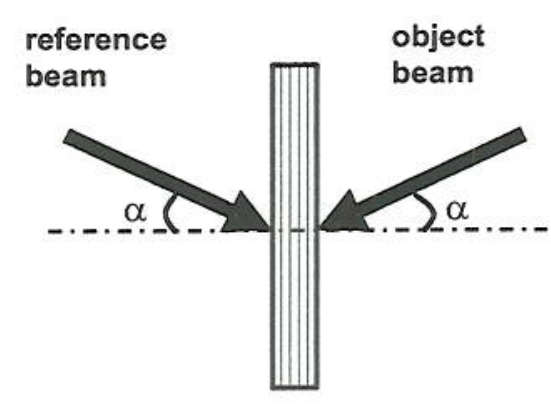

a)

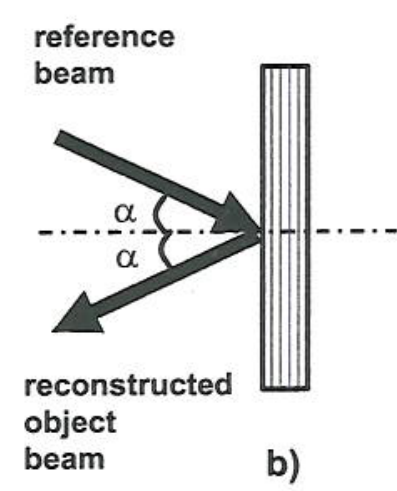

b)

Fig. 1 Recording of a reflection hologram a) and reconstruction of the object beam b).

If the reflection hologram is illuminated with one of the recording beams (Fig. 1b) it will diffract the light to produce the other recording beam. If the hologram is illuminated with white light at a range of incident angles it will reconstruct a beam having a wavelength range satisfying the relation (1). As the spectral selectivity of the reflection hologram is very high, especially when the thickness of the hologram is high enough, the observer will see a specific colour, essentially 
that of the recording beam. If one of the recording beams is a complex wavefront coming from a three dimensional object, the recorded reflection hologram will have the property that it can reconstruct this wavefront when illuminated with white light. This means that any distant point source of light will produce, through diffraction, a three dimensional image of the original object in a colour determined by the wavelength of recording beam. This assumes that the dimensions of the hologram are the same as they were during the recording.

\subsection{Principle of operation of the humidity indicator}

Figure 2 can be used to explain the basic principle of operation of the holographic humidity indicator described in this paper. A reflection holographic optical element is recorded in photopolymer recording material. When illuminated with white light the device will reflect the light in a narrow spectral range. As a result the observer sees a specific colour which depends also on the holographic fringe spacing determined by relation (1). If the holographic optical element is disposed in a medium that can easily absorb or release water as a result of increase or decrease of the relative humidity of the environment and consequently can easily swell or shrink, these dimensional changes will lead to change in the fringe spacing. The ultimate effect is a change of the observed colour of the diffracted light as a result of change of the relative humidity.

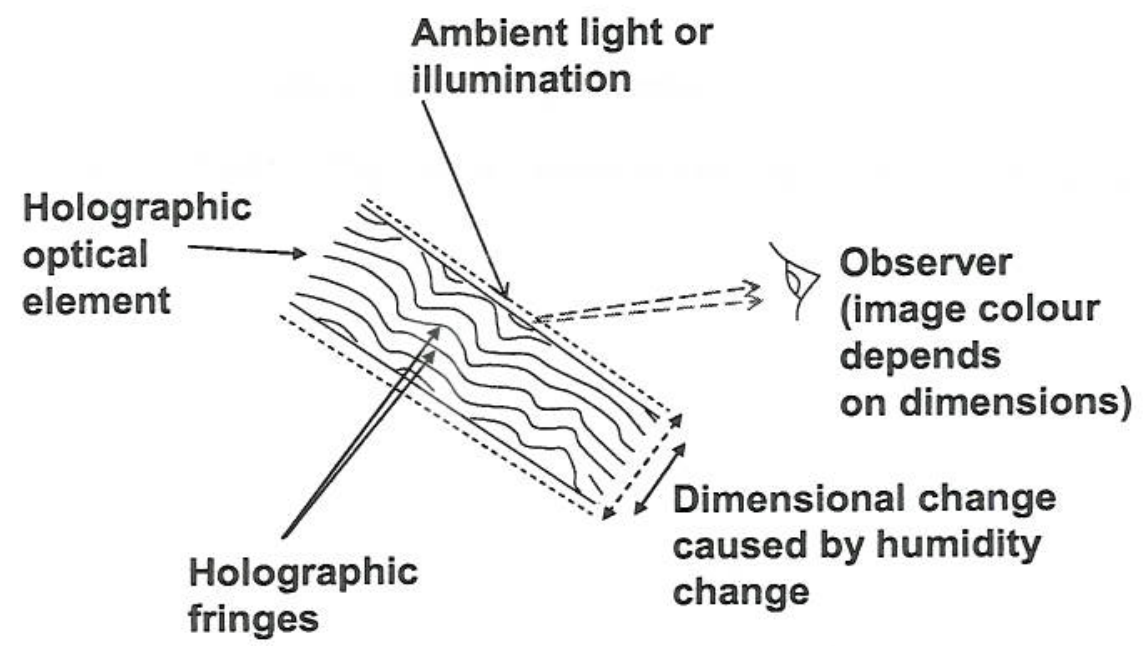

Fig. 2 Principle of operation of the holographic humidity indicator.

\section{EXPERIMENTAL}

\subsection{Photopolymer layer preparation}

The photopolymer material used in this study is a self-processing acrylamide based photopolymer developed at the Centre for Industrial and Engineering Optics, Dublin Institute of Technology ${ }^{6-8}$. The photosensitive layer consists of two monomers - acrylamide and N,N Methylene bisacrylamide, a polymeric binder - polyvinyl alcohol, a photosensitiser Erythrosin B and an initiator triethanolamine (TEA). Different mixtures of these components were studied in order to obtain optimum diffraction efficiency and optimum humidity response of the reflection holographic optical element. The preparation of one example that has been studied is described below. Two $\mathrm{ml}$ of triethanolamine was added to $17.5 \mathrm{ml}$ stock solution of polyvinyl alcohol (PVA) $(10 \% \mathrm{w} / \mathrm{v})$. Then the monomer, $0.6 \mathrm{~g}$ acrylamide and the crosslinker, $0.2 \mathrm{~g}$ of N,N Methylene bisacrylamide, were added. Finally, $4 \mathrm{ml}$ of Erythrosin B dye was added from a stock solution with 
concentration of $1.1 \mathrm{mM}$. $1.5 \mathrm{ml}$ of this solution was spread on $50 \times 50 \mathrm{~mm}$ plate. The samples were dried for $24-36$ hours. When the dependence of the humidity response on the hologram thickness was studied the layers were prepared from different amounts of photopolymer solution varying from 0.5 to $2 \mathrm{ml}$ per $50 \times 50 \mathrm{~mm}$ slide.

The thickness of the layers was measured by cutting the photopolymer layer using a sharp scalpel and measuring the dimensions of the cut with a white light interferometric (WLI) surface profiler MicroXAM S/N 8038 .

\subsection{Recording of the holographic optical element}

The reflection gratings were recorded using the recording geometry shown in Fig.1a. The frequency doubled output from $\mathrm{NdYVO}_{4}$ laser (Verdi 05) was used to record the holograms at wavelength of $\lambda=532 \mathrm{~nm}$. Due to the high sensitivity of the material the total exposure of recording did not exceed $100 \mathrm{~mJ} / \mathrm{cm}^{2}$. Some of the humidity indicators incorporating a holographic image of an object were recorded as Denisyuk type holograms.

\subsection{Measurement of the spectral response}

A controlled environment chamber with humidity control system was purchased from Electro-tech systems, inc. The system model 503-20 consists of a glove box, a microprocessor controller, a dehumidification system and an ultrasonic humidification system. The relative humidity in the chamber can be maintained to better than $\pm 1 \% \mathrm{RH}$ of the set point. The operational range is from $5 \%$ to $100 \% \mathrm{RH}$. A fast responding capacitive type sensor providing measurement accuracy of $\pm 2 \% \mathrm{RH}$ over the range of $0-90 \%$ and $3 \%$ from 90 to $100 \% \mathrm{RH}$ is incorporated in the chamber.

The test optical set-up was assembled in the humidity chamber. The light from an AvaLight-HAL-S light source was used to probe the reflection holograms. In order to guide the light into the humidity chamber, the light was coupled to a fibre and then collimated by a lens. The reflected light from the probed hologram was then coupled to another fibre with the help of a second lens and then guided to a spectral analyser Model AvaSpec-2048. In order to obtain the calibration curve for a specific device the relationship between the wavelength corresponding to the maximum intensity in the spectral response of the hologram and the relative humidity in the chamber was determined.

When the visual colour change of the sample was studied, the hologram was illuminated by a white light spot lamp and the holographic image was recorded by a digital camera model Sony Cyber-shot DSC300.

\subsection{Measurement of the time response}

Measurement of the response time of the humidity indicator was carried out by recording the change of the position of the maximum intensity in the spectral response of the hologram at different times after achieving preset humidity. The increment in humidity was $10 \%$. The time for achieving the preset humidity was less than $1 \mathrm{~min}$. The change in the peak position during this latter time interval for layers with thickness above $30 \mu \mathrm{m}$ was negligible. Only the changes after achieving the preset humidity were taken into account.

\section{RESULTS AND DISCUSSION}

\subsection{Spectral response of the recorded reflection holographic gratings}

The typical spectral response of a reflection holographic grating recorded in a $30 \mu \mathrm{m}$ thick photopolymer layer is presented in Fig. 3. As expected from such a holographic optical element ${ }^{4}$ it is very selective with respect to the wavelength of the reflected light - the full width at the half maximum is only $2.8 \mathrm{~nm}$. This explains the single colour 
appearance of the reconstructed image in Fig. 4. The position of the peak in the spectral response curve can be easily determined from these data and its change with the change of the relative humidity in the controlled environment can be easily followed in order to calibrate the humidity indicator.

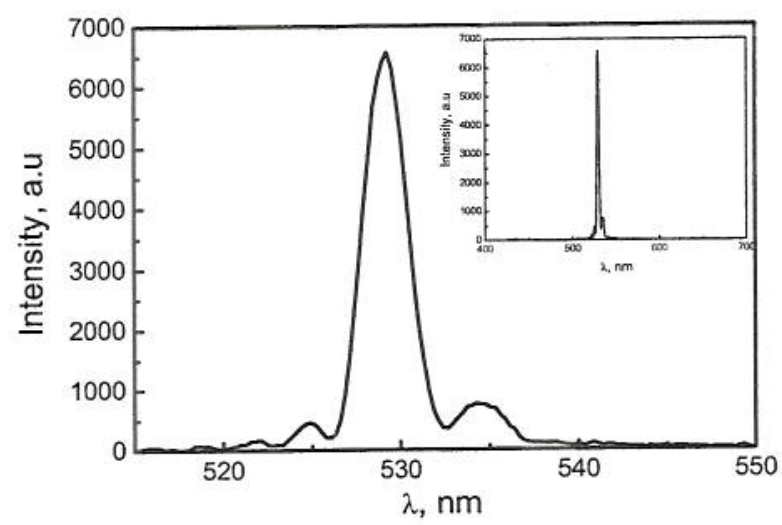

Fig. 3 Spectral response of reflection holographic grating recorded in $30 \mu \mathrm{m}$ thick layer.

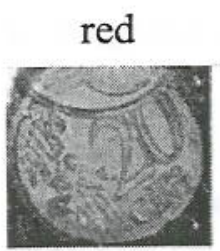

RH $50 \%$ green

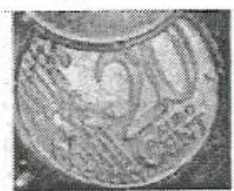

$\mathrm{RH} 40 \%$ blue

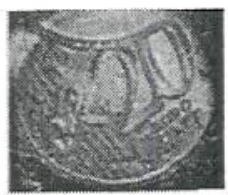

RH 20\%

Fig. 4 Colour appearance of a hologram exposed to different humidity.

\subsection{Humidity response of the reflection hologram}

An example of the humidity response of a reflection hologram recorded in a photopolymer layer is shown in Fig. 5. It is seen that when the relative humidity changes from $7 \%$ to $70 \%$ the position of the peak in the hologram spectral response also changes by $100 \mathrm{~nm}$. With increasing humidity the position of the spectral peak shifts towards the red region of the spectrum. This could be readily explained by the swelling of the photopolymer layer and the consequent increase of the holographic fringe spacing. When the relative humidity decreases, the spectral peak shifts towards the blue region of the spectrum and this could be explained by shrinkage of the photopolymer layer and decrease of the holographic fringe spacing. It should be noticed that the relation between the position of the spectral peak and the value of the relative humidity is nonlinear. The humidity indicator is more sensitive at higher than at lower relative humidity. 


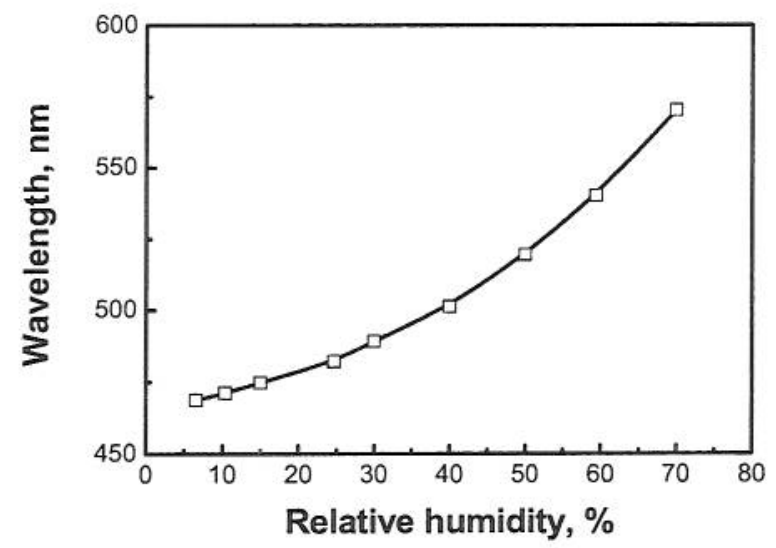

Fig. 5 Humidity response of a reflection hologram recorded in $30 \mu \mathrm{m}$ thick photopolymer layer.

\subsection{Effect of humidity of recording on the calibration curve}

It was observed that the relative humidity in the laboratory during the recording process is important for the final colour appearance of the hologram and also influences the calibration curve. For instance two different humidity indicators were recorded at relative humidity values of $38 \%$ and $29 \%$ respectively. The calibration curves of these two indicators are shown in Fig. 6.

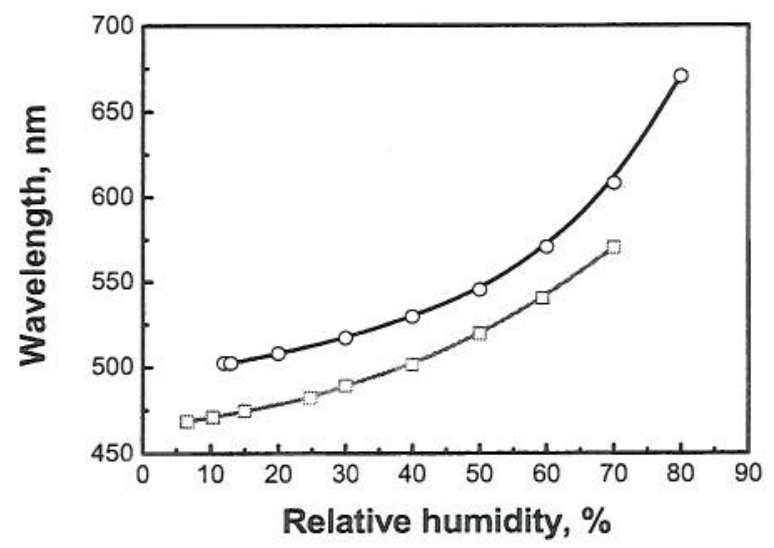

Fig. 6 Calibration curves for humidity indicators recorded at $29 \% \mathrm{RH}$ (black) and $38 \% \mathrm{RH}$ ( grey).

There are two observations that are worth noting. Firstly both calibration curves are shifted so that the wavelength of recording $532 \mathrm{~nm}$ is observed at higher humidity than the humidity of recording. This implies that the photopolymer layers have shrunk during or immediately after the recording and, when exposed to the same humidity as the humidity of recording, the replayed wavelength is blue shifted. In order to replay the original wavelength one needs to expose the layers to higher than the humidity of recording. The second observation is that changing the relative humidity at the recording stage produces a different colour appearance of the humidity indicator at a given value of relative humidity. This provides an additional tool for modification of the calibration curve. 


\subsection{Reversibility}

The other important aspect related to the operation of the holographic humidity indicator was its reversibility. Preliminary studies have been carried out showing excellent reversibility, though the number of cycles tested is still low.

In order to study the reversibility of the indicator it was cycled anumber of times initially from 30 to $70 \% \mathrm{RH}$ and from 20 to $80 \% \mathrm{RH}$. The results from the first study are presented in Fig. 7. It is seen that after four cycles the change in the position of the spectral peak is in order of $1 \mathrm{~nm}$. Such change in the actual appearance of the hologram could not be detected by eye. Similarly, when the appearance of the holographic image of a coin exposed to 7 cycles from 20 to $80 \% \mathrm{RH}$ was observed no irreversible change was detected. Further studies are underway looking at a larger number of cycles.

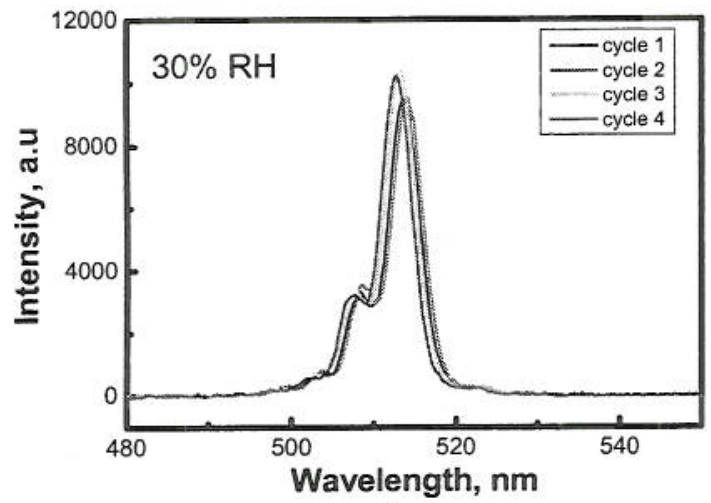

a)

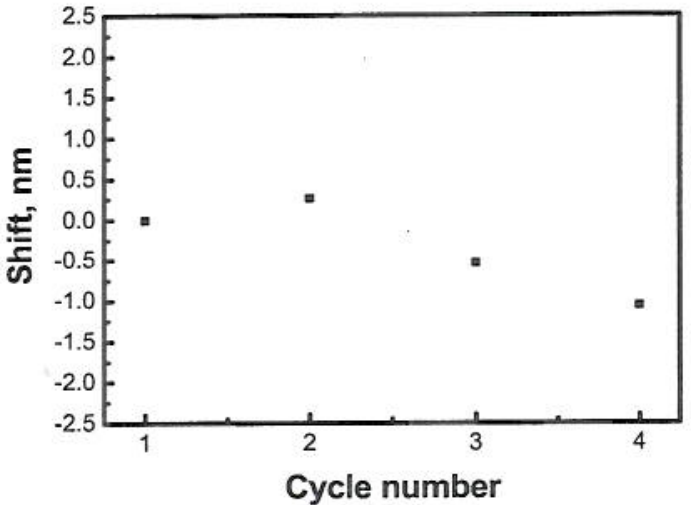

b)

Fig. 7 Spectral response of a humidity indicator at $30 \% \mathrm{RH}$ after it has been exposed to $70 \% \mathrm{RH}$ during several cycles a) and deviation of the spectral peak from its initial position after each cycle b).

\subsection{Time response}

Another important characteristic of the humidity indicator is its time response. Using the methodology described in the experimental section we have measured the time response of samples recorded in photopolymer layers with different thicknesses. The data for 30 and $90 \mu \mathrm{m}$ thick layers are shown in Fig. 8. As can be seen from the figure the thickness of the layers has a significant influence on the time required to achieve the final appearance at specific relative humidity. For the thinner layer $10 \mathrm{~min}$ were required to reach a fixed colour at $60 \% \mathrm{RH}$ and for the thicker one as much as $50 \mathrm{~min}$ were necessary.

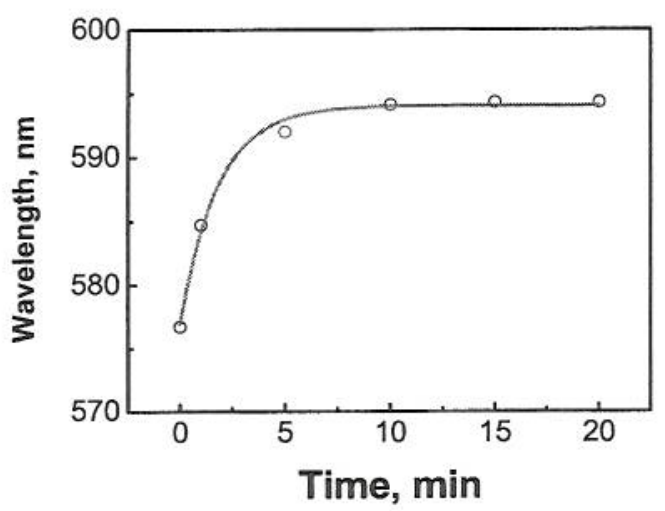

a)

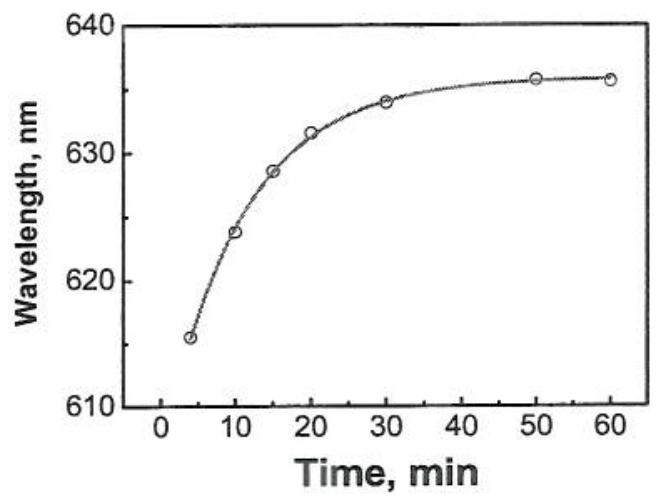

b)

Fig. 8 Time response of samples of $30 \mu \mathrm{m}$ a) and $90 \mu \mathrm{m}$ thickness b). The change of the spectral peak position was followed after the relative humidity was changed from 50 to $60 \% \mathrm{RH}$. 


\subsection{Effect of exposure to elevated temperatures}

In order to explore different methods for modification of the operational range of the humidity indicator we have studied the effect of exposure to elevated temperature of a previously recorded humidity indicator. An example is presented in Fig. 9. It is seen that the heating of the sample causes a shift in the calibration curve. This is probably due to irreversible shrinkage caused by the exposure to the temperature of $125^{\circ} \mathrm{C}$. This shift in the calibration curve can be used to adjust the exact colour of the hologram at a specific humidity. It can also be used to make the hologram invisible at a specific humidity, as it will reconstruct the image in the UV spectral range, and appear only when exposed to a higher humidity level.

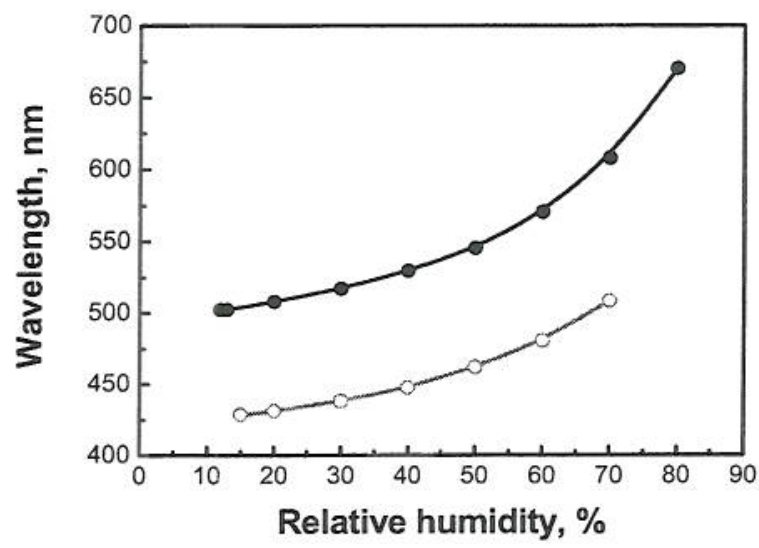

Fig. 9 Influence of exposure to temperature of $125^{\circ} \mathrm{C}$; before heating (black) and after heating (grey).

\section{CONCLUSSIONS}

In conclusion, we have designed, fabricated and characterised a photopolymer based holographic humidity indicator. The device is reversible, has accuracy better than $5 \% \mathrm{RH}$ and a range of response times that can be controlled by the thickness of the photopolymer layer used to record the holographic humidity indicator. The operational range of the device can be modified by recording the holograms at different relative humidity as well as by exposing the already recorded holograms to elevated temperatures.

\section{REFERENCES}

1. A.J. Marshall, J. Blyth, C. Davidson, C.R. Lowe, "PH-sensitive holographic sensors", Analyt. Chem., 75 (17), 4423-4431, 2003.

2. S. Kabilan, A.J. Marshall, F.K. Sartain, M.C. Lee, A. Hussain, X.P. Yang, J. Blyth, N. Karangu, K. James, J. Zeng, D. Smith, A. Domschke, C.R. Lowe, "Holographic glucose sensors", Biosensors \& Bioelectronics, 20 (8), $1602-1610,2005$.

3. C.R. Lowe, M.R. Bradley, J. Blyth, A. G. Mayes, "Hologram used us a sendor", patent WO95/26499, 1995.

4. P. Hariharan, Optical Holography, Cambridge Studies in Modern Optics, Cambridge University Press, 1996.

5. H. Kogelnik, "Coupled wave theory for thick hologram gratings", Bell Syst. Tech. J. 48, 2909-2947, 1969.

6. S. Martin, C.A. Feely and V. Toal, "Holographic recording characteristics of an acrylamide-based photopolymer", Appl. Opt., 36, 5757-5768, 1997.

7. I. Naydenova, R. Jallapuram, S. Martin, R. Howard, V. Toal, Appl. Opt, 43 (14) 2900-05, 2004.

8. S. Martin, I. Naydenova, V. Toal, R. Jallapuram, R. G. Howard, "Two way diffusion model for the recording mechanism in a self developing dry acrylamide photopolymer", Proceedings of SPIE, 6252, 625205, 2006. 